\title{
Can Implantable Hearing Devices be a Solution for Michel's Deformity? A Case Report
}

\author{
Mrs Maya Varma $\mathbf{R}^{1 *}$, Remya Ramachandran ${ }^{2}$ and Sudharma B, Medikeri's ${ }^{3}$ \\ ${ }^{1}$ Department of Speech and Hearing Sciences, School of Allied Health Sciences, Manipal Academy of Higher Education, Manipal, India \\ ${ }^{2}$ Sree Chitra Tirunal Institute for Medical Sciences and Technology, Kerala, India \\ ${ }^{3}$ Superspeciality ENT Center, Medikeri, Bangalore, India
}

Submission: October 29, 2018; Published: November 09, 2018

*Corresponding author: Mrs Maya Varma R, Department of Speech and Hearing Sciences, School of Allied Health Sciences, Manipal Academy of Higher Education, Manipal, India

Abstract

Objective: The aim of the present study is to highlight on the limited rehabilitation options and importance of early identification and intervention on a child with unilateral Michel's deformity.

Method: Detailed radiological evaluation, audiological evaluation and hearing aid trail were done. Detailed speech-language assessment was done during first visit, after six months and one year.

Results: Radiological evaluation confirmed Michel's deformity. A detailed audiological evaluation shows that right ear hearing sensitivity within normal limits and left ear moderately severe to severe sensorineural hearing loss. Child was not benefitting with hearing aids. Speech and language assessment shows delay in expressive language skills and but follow-up after intervention shows age adequate language skills.

Conclusion: An early identification and intervention of unilateral Michel's deformity can provide better communication skill. In cases of Michel'sdeformitythe rehabilitative options are highly limited and this study sheds light to the future development.

Keywords: Michel's deformity; Cochlear implantation; Vibrotactile devices

\section{Introduction}

Congenital sensorineural hearing loss (SNHL) can be unilateral or bilateral[1]. Congenital unilateral sensorineural hearing loss (USNHL) can be due to the malformation of the inner ear caused due to a genetic factors or idiopathic[2]. Most of the inner ear malformations arise when formation of the membranous labyrinth is interrupted during the first trimester of pregnancy[3]. Michel (1863) reported that complete labyrinthine aplasia or Michel's deformity is the most that the most severe form of inner ear malformation. It can be unilateral or bilateral, in which unilateral is very rare. It is characterized by undifferentiated inner ear structures and it will be an absolute contradiction for cochlear implantation on the affected side[4]. Therefore, the aim of the present study is to highlight on the limited rehabilitation options and importance of early identification and intervention on a child with unilateral Michel's deformity.

\section{Method}

\section{Participant}

A five months old boy reported to a tertiary care hospital when the parents noticed a facial deviation to the right side and difficulty to open right eye. The Department of Paediatrics
Neurology evaluated and discharged the child by giving steroids for four months. Child had a complete recovery within three months and was asymptomatic till one and a half-year old. Second episode of facial nerve palsy occurred at the age of one and a half-year old and child was admitted for detailed investigation.

\section{Procedure}

Radiological evaluations were done using Magnetic Resonance Imaging (MRI) and Computer Tomography (CT). After radiological evaluation the child was referred to Department of Speech Pathology and Audiology for detailed audiological evaluation.Immittance audiometry was performed using calibrated Grason Stadler Incorporates (GSI) Tympstar equipment Version:1. Tympanograms and acoustic reflex thresholds were obtained using a $226 \mathrm{~Hz}$ probe tone frequency with a pump rate of 50 dapa. Eletrophysiological testing was performed in a sound treated room using Intelligent Hearing Screening (IHS) Smart EP Software, Version: 3.94 including Distortion Product Otoacoustic Emission (DPOAE), Auditory Brainstem Response (ABR) and Auditory Steady State Responses (ASSR). The DPOAE signal to noise ratio estimation was done at frequencies of $598 \mathrm{~Hz}, 691 \mathrm{~Hz}, 785 \mathrm{~Hz}, 902 \mathrm{~Hz}, 1031 \mathrm{~Hz}, 1184$ 
$\mathrm{Hz}, 1371$ Hz, 1594 Hz, 1816 Hz, 2098 Hz, 2402 Hz, 2754 Hz, 3152 $\mathrm{Hz}, 3621 \mathrm{~Hz}, 4184 \mathrm{~Hz}, 4816 \mathrm{~Hz}, 5496 \mathrm{~Hz}, 6340 \mathrm{~Hz}$ and $7277 \mathrm{~Hz}$. Threshold estimation was done using a click stimulus with a repetition rate of $11.1 / \mathrm{sec}$. The electrode sites were cleaned using NuPrep skin preparation gel to bring the impedance within acceptable limit and a Ten 20 conductive gel was used for creating a favoring condition for the electrodes to pick the responses from the skin. Etymotic ER-3 earphones were used for stimulus presentation and gold cup electrodes were employed. ASSR was performed using the default settings and stimulus was delivered using the same transducers as of BERA.

\section{Speech and Language assessment and Intervention}

A detailed speech and language assessment was carried including details of birth history, developmental history and speech and language development. Case history shows that there was no significant birth history, age appropriate motor development and social interaction skills. Speech and language evaluation was done by observing the child during an interaction session with the clinician, mother and other family members. Formal language assessment was done using Receptive Expressive Emergent Language Scale (REELS) - 3. The child was enrolled for speech and language intervention for six days per week with each session extending upto one hour. The goals taken were to work upon the expressive language skills. Speech and Language skills were re-evaluated after six months and one year after the initiation of therapy.

\section{Results}

MRI findings showed Internal Auditory Meatus (IAM), cochlea and semicircular canal (SCC) appear normal on the right side. On the left side VII and VIII cranial nerve appear hypoplastic as compared to right side. The nerves exist the temporal bone though a foreman (IAM) and is placed further caudally, compared to the IAM of right side. On the left side cochlea and SCC canal is not visualized separately and modulus is malformed. MRI results were summarized as hypoplastic VII and VIII cranial nerve with cochlear malformation indicating Michel's deformity. A temporal bone CT scan shows that left side cochlear and vestibule canal not visualized separately and appears as a single cavity, severe stenosis of IAM, facial canal at the descending mastoid part appear shortened. Temporal bone CT scan findings suggested left inner ear malformation with hypoplasia of SCC and common cavity for cochlear and vestibule. Detailed audiological evaluation shows bilateral 'A' type tympanogram indicating a normal conductive pathway with right ear ipsilateral acoustic reflexes present, right ear contralateral acoustic reflexes absent and left ear ipsilatral and contralateral acoustic reflexes absent, DPOAE was present in the right ear indicating of normal outer hair cell $(\mathrm{OHC})$ functioning and left ear DPOAE was absent indicating of $\mathrm{OHC}$ dysfunctioning, $\mathrm{ABR}$ in the right ear shows Vth peak was present till $30 \mathrm{dBnHL}$ with good morphology, amplitude and replicability and left ear Vth peak was absent till
99 dBnHL with poor morphology, amplitude and replicability, ASSR shows that right ear hearing sensitivity was within normal limits and left ear moderately severe to severe hearing loss. Detailed audiological test battery approach indicated that right ear hearing sensitivity was within normal limits and left ear moderately severe to severe sensorineural hearing loss. Unilateral (left) Hearing Aid Trail (HAT) was done using BernafonInizia 1 Receiver In the Ear (RITE) with power receiver in a sound treated room. Child's responses were observed by occluding the right ear with a hard mould without vent. There was no significant different in the responses with and without HA and parents were counseled regarding the same.Speech and language assessment using REELS- 3 was done during the first visit shows Reception Language Age (RLA) was 16 to 18 months (age adequate) and Expressive Language Age (ELA) was delayed to eight to nine months. Based on the complete speech, language and hearing evaluation the child was diagnosed as expressive language delay with unilateral sensorineural hearing loss associated with Michel's deformity. A follow-up after six months and one years shows that the child's receptive and expressive language was age adequate and child's performance at school was good as reported by the teachers and parents. The parents were also counselled regarding the need of speech and language stimulation and limited amplification options for the left ear hearing loss and importance of preserving hearing in the right ear.

\section{Discussion}

Congenital malformation of inner ear and hearing loss is always a challenging factor for medical, speech language pathologist, audiologist and parents. Among this according to Jackler et al. [3] bony malformation including Michel's deformity or complete labyrinthine aplasia is one of the severe forms of congenital inner ear malformation. Literature shows that Michel's anomaly occur due to the arrest of otic placode early in the 3rd week of gestational age[5]. Radiological studies in congenital inner ear malformation show that in Michel's deformity facial nerve palsy is mostly a concomitant condition. Autopsy findings suggest that facial nerve is normally developed but may follow an abnormal course. IAM is either absent or very small if it carriers the facial nerve. Facial nerve may penetrate directly into the temporal bone passing into middle ear through small IAM. In Michel's anomaly neither the labyrinthine portion of facial nerve nor pyramidal eminence is developed. The developmental arrest of the optic placodes of the second brachial arches will lead to inner ear anomalies; however, normal functioning of the facial nerve is independent of the development of inner ear[6]. In the present case facial nerve, exist temporal bone through IAM and is placed caudally. This indicates that the development of facial nerve was normal and function could be affected due to the hypoplasia of IAM, but however the patient recovered from facial nerve palsy and is symptomatic after the medical management. 
The severe inner ear anomaly of Michel's deformity including incompletely developed SCC, vestibule and common cavity had contributed to the congenital hearing loss on the left ear in the present case study. Congenital malformation, such as Mondini may benefit from cochlear implants [7]. However, in Michel's deformity absence of inner ear development rules out the possibilities of cochlear implantation. In the present, cochlear implantation was not suggested because of the absence of inner ear, normal hearing of right ear and normal speech and language skills attained by intervention. The child had a speech and language delay due to lack of stimulation. Optimal identification and rehabilitation of the child's auditory and speech-language skills and adequate parental counseling and awareness have improved the child communication skills.

\section{Conclusion}

Inner ear malformation with severe dysplasia is a challenging factor of any medical or rehabilitative audiologist to decide the appropriate rehabilitative option. The present study has rule out the possibility of any audiological rehabilitation. Surprisingly literature has reported that auditory brainstem implant (ABI) and vibrotactile devices can be profitable for Michel's deformity [8]. Therefore, it is highly warranted that the future researches who is working closely to improve technological based advancement in the field of audiological rehabilitation to find some possible solution for such conditions.

\section{References}

1. YiinRSZ,Tang PH, Tan TY (2011) Review of congenital inner ear abnormalities on CT temporal bone. Br J Radiol84(1005):859-863.

2. Joshi VM, Navlekar SK, Kishore GR, Reddy KJ, Kumar EC (2012) CT and MR imaging of the inner ear and brain in children with congenital sensorineural hearing loss. Radiographics32(3):683-698.

3. JacklerRK, LuxfordWM, HouseWF (1987) Congenital malformations of the inner ear: a classification based on embryogenesis. Laryngoscope97(4): 2-14.

4. AmarnathC, SathyanG, SoniyaR, PeriakaruppanAL, ShankarKS (2016) Evaluation of embryological sequences of ear anomalies and its radiological relevance. Indian Journal of Otology 22(4): 248.

5. Dupuch MK, Brito DA, GhasliK, ChouardCH (1999) CT and MR findings of Michel anomaly: Inner ear aplasia. AJNR Am J Neuroradiol20(2): 281-284.

6. PoojaVD, ShaileshkumarGS, DeepakVD, BushraRB, NiravTR, et al. (2015) High-resolution computerizedtomography and Magnetic Resonance Imaging (MRI) in preoperative evaluation of cochlear implant patients. Advanced Arab Academy of Audio-Vestibulogy Journal2(1): 1-4.

7. AhmadRL, LokmanS (2005) Cochlear implantation in congenital cochlear abnormalities. MedJMalaysia 60(3): 379-382.

\section{Your next submission with Juniper Publishers will reach you the below assets}

- Quality Editorial service

- Swift Peer Review

- Reprints availability

- E-prints Service

- Manuscript Podcast for convenient understanding

- Global attainment for your research

- Manuscript accessibility in different formats

( Pdf, E-pub, Full Text, Audio)

- Unceasing customer service

Track the below URL for one-step submission https://juniperpublishers.com/online-submission.php 